Published in final edited form as:

Pediatr Infect Dis J. 2008 September ; 27(9): 808-814. doi:10.1097/INF.0b013e31817109a4.

\title{
Morbidity and Mortality Among a Cohort of Human Immunodeficiency Virus Type 1-Infected and Uninfected Pregnant Women and Their Infants From Malawi, Zambia, and Tanzania
}

\author{
David Chilongozi, CO, MPH ${ }^{\star}$, Lei Wang, $\mathrm{PhD}^{\dagger}$, Lillian Brown, MPH ${ }^{\ddagger}$, , Taha Taha, MD, PhD $\|$,

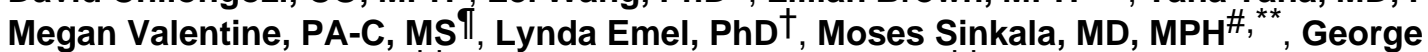 \\ Kafulafula, MBBS, FCOG ${ }^{\dagger}$, Ramadhani A. Noor, MD, MPH $\neq \ddagger$, Jennifer S. Read, MD, MS, MPH, \\ DTM\&H, MS§§, Elizabeth R. Brown, ScD \|\| , Robert L. Goldenberg, MD ${ }^{\star \star}$, and Irving Hoffman, \\ PA, MPH $\S$ for the HIVNET 024 Study Team \\ *University of North Carolina Project, Lilongwe, Malawi \\ †Statistical Center for HIV/AIDS Research and Prevention, Fred Hutchinson Cancer Research \\ Center, Seattle, WA \\ FUniversity of North Carolina School of Public Health, University of North Carolina, Chapel Hill, NC \\ §Division of Infectious Diseases, University of North Carolina, Chapel Hill, NC \\ "Bloomberg School of Public Health, Johns Hopkins University, Baltimore, MD \\ IFamily Health International, Research Triangle Park, NC \\ \#Centre for Infectious Disease Research in Zambia, Lusaka, Zambia \\ *Department of Obstetrics and Gynecology, University of Alabama, Birmingham, AL \\ ttDepartment of Obstetrics and Gynaecology, College of Medicine, University of Malawi, Blantyre, \\ Malawi
}

\footnotetext{
Copyright $(9) 2008$ by Lippincott Williams \& Wilkins

Address for correspondence: Irving Hoffman, PA, MPH, Division of Infectious Diseases, University of North Carolina at Chapel Hill, 130 Mason Farm Rd. Chapel Hill, North Carolina 27599. E-mail: hoffmani@ med.unc.edu.

HIVNET 024 Team: Protocol Co-Chairs: Taha E. Taha, MD, PhD (Johns Hopkins University Bloomberg School of Public Health); Robert Goldenberg, MD (University of Alabama at Birmingham); In-Country Co-Chairs/Investigators of Record: Newton Kumwenda, $\mathrm{PhD}$, George Kafulafula, MBBS, FCOG (Blantyre, Malawi); Francis Martinson, MD, PhD (Lilongwe, Malawi); Gernard Msamanga, MD, ScD (Dare es Salaam, Tanzania); Moses Sinkala, MD, MPH, Jeffrey Stringer, MD (Lusaka, Zambia); U.S. Co-Chairs: Irving Hoffman, PA, MPH (University of North Carolina, Chapel Hill); Wafaie Fawzi, MD, DrPH (Harvard School of Public Health); InCountry Investigators, Consultants and Key Site Personnel: Robin Broadhead, MBBS, FRCP, George Liomba, MBBS, FRCPath, Johnstone Kumwenda, MBChB, MRCP, Tsedal Mebrahtu, ScM, Pauline Katunda, MHS, Maysoon Dahab, MHS (Blantyre, Malawi); Peter Kazembe, MBChB, David Chilongozi CO, MPH, Charles Chasela CO, MPH, George Joaki, MD, Willard Dzinyemba, Sam Kamanga (Lilongwe, Malawi); Elgius Lyamuya, MD, PhD, Charles Kilewo, MD, MMed, Karim Manji, MD, MMed, Sylvia Kaaya, MD, MS, Said Aboud, MD, MMed, Muhsin Sheriff, MD, MPH, Elmar Saathoff, PhD, Priya Satow, MPH, Illuminata Ballonzi, SRN, Gretchen Antelman, ScD, Edgar Basheka, BPharm (Dar es Salaam, Tanzania); Victor Mudenda, MD, Christine Kaseba, MD, Maureen Njobvu, MD, Makungu Kabaso, MD, Muzala Kapina, MD, Anthony Yeta, MD, Seraphine Kaminsa, MD, MPH, Constantine Malama, MD, Dara Potter, MBA, Maclean Ukwimi, RN, Alison Taylor, BSc, Patrick Chipaila, MSc, Bernice Mwale, BPharm (Lusaka, Zambia); U.S Investigators, Consultants and Key Site Personnel: Priya Joshi, BS, Ada Cachafeiro, BS, Shermalyn Greene, PhD, Marker Turner, BS, Melissa Kerkau, BS, Paul Alabanza, BS, Amy James, BS, Som Siharath, BS, Tiffany Tribull, MS (UNC-CH); Saidi Kapiga, MD, ScD, George Seage, PhD (HSPH); Sten Vermund, MD, PhD, William Andrews, PhD, MD, Deedee Lyon, BS, MT(ASCP) (UAB); NIAID Medical Officer: Samuel Adeniyi-Jones, MD; NICHD Medical Officer: Jennifer S. Read, MD, MS, MPH, DTM\&H; Protocol Pharmacologist: Scharla Estep, RPh, MS; Protocol Statisticians: Elizabeth R. Brown, ScD, Thomas R. Fleming, PhD, Anthony Mwatha, MS, Lei Wang, PhD, Ying Q. Chen, PhD; Protocol Virologist: Susan Fiscus, PhD; Protocol Operations Coordinator: Lynda Emel, PhD; Data Coordinators: Debra J. Lands, Ed.M, Ceceilia J. Dominique; Systems Analyst Programmers: Alice H. Fisher, BA, Martha Doyle; Protocol Specialist: Megan Valentine, PA-C, MS.
} 


\section{¥¥Muhimbili University, Dar-Es Salaam, Tanzania \\ $\S \S$ Pediatric, Adolescent, and Maternal AIDS Branch, National Institute of Child Health and Human Development, University of Washington, Seattle, WA.}

"IIDepartment of Biostatistics, University of Washington, Seattle, WA.

\section{Abstract}

Background: Morbidity and mortality patterns among pregnant women and their infants (before antiretroviral therapy was widely available) determines HIV-1 diagnostic, monitoring, and care interventions.

Methods: Data from mothers and their infants enrolled in a trial of antibiotics to reduce mother-tochild-transmission of HIV-1 at 4 sub-Saharan African sites were analyzed. Women were enrolled during pregnancy and follow-up continued until the infants reached 12 months of age. We describe maternal and infant morbidity and mortality in a cohort of HIV-1-infected and HIV-1-uninfected mothers. Maternal and infant factors associated with mortality risk in the infants were assessed using Cox proportional hazard modeling.

Results: Among 2292 HIV-1-infected mothers, 166 (7.2\%) had a serious adverse event (SAE) and $42(1.8 \%)$ died, whereas no deaths occurred among the 331 HIV-1 uninfected mothers. Four hundred twenty-four (17.8\%) of 2383 infants had an SAE and 349 (16.4\%) died before the end of follow-up. Infants with early HIV-1 infection (birth to $4-6$ weeks) had the highest mortality. Among infants born to HIV-1-infected women, maternal morbidity and mortality $(P=0.0001)$, baseline CD4 count $(P=0.0002)$, and baseline plasma HIV-1 RNA concentration $(P<0.0001)$ were significant predictors of infant mortality in multivariate analyses.

Conclusions: The high mortality among infants with early HIV-1 infection supports access to HIV-1 diagnostics and appropriate early treatment for all infants of HIV-1-infected mothers. The significant association between stage of maternal HIV-1 infection and infant mortality supports routine CD4 counts at the time of prenatal HIV-1 testing.

\section{Keywords}

HIV-1 infection; infant mortality; maternal morbidity and mortality; sub-Saharan Africa; pregnant women

The HIV-1 epidemic in sub-Saharan Africa continues to expand and affect women and children disproportionately. ${ }^{1}$ In recent years, with support from a variety of donor organizations, including the Global Fund on AIDS, Tuberculosis and Malaria, and the President's Emergency Plan For AIDS Relief (PEPFAR), significant progress has been made in increasing the availability of antiretroviral therapy (ART) and the number of prevention of mother-to-child transmission (PMTCT) programs. ${ }^{2,3}$ However, for a variety of logistical, financial, and socio/ cultural reasons, these programs are not covering all in need. In Malawi, for example, there are now more than 100,000 persons who have initiated ART since December 2003. ${ }^{4}$ However, it is predicted that 1 million are infected with HIV, 200,000 require ART, and each year another 81,000 become infected and will need ART in the coming years. ${ }^{4}$ Many HIV-1-infected adults and children in this region of the world still go undiagnosed, or even if diagnosed, are often lost to the health care system and present for care too late even for ART to help. 5,6

From mid-2001 to early-2003, a multisite clinical trial (HIVNET 024) took place in Malawi, Tanzania, and Zambia to evaluate the efficacy of antibiotics for prevention of chorioamnionitisrelated mother-to-child transmission (MTCT) of HIV-1 ${ }^{7,8} \mathrm{HIV}-1$-infected and uninfected pregnant women were enrolled in their second trimester and followed periodically, along with their infants, for 12 months after delivery. The study began before ART was readily available 
in these countries. The routine provision of nevirapine prophylaxis for PMTCT during the conduct of this trial was among the first utilizations of the HIVNET 012 findings in the region. 8

As part of the safety objectives of this study, and in accordance with Good Clinical Practice (GCP), all serious adverse events (SAEs), including all maternal and infant deaths were documented and reported. Thus, this study is one of the last controlled trials in sub-Sahara Africa that documents the natural history of HIV-1 disease for pregnant women and their newborns.

Many gaps remain in HIV-1 care programs in sub-Saharan Africa, particularly in the areas of early diagnosis, ART access, and CD4 monitoring. We undertook this tertiary analysis from this large clinical trial to determine the morbidity and mortality patterns among African women and infants from the second trimester of pregnancy among women and among the mother infant pairs from delivery to 12 months postpartum. Knowledge of these factors might inform ART treatment and care programs, particularly the time frame when HIV-1-infected pregnant women and their newborns would benefit the most from HIV-1 diagnostic, monitoring, and care interventions.

\section{METHODS \\ HIVNET 024 Trial}

We conducted an observational cohort analysis from HIVNET 024, a randomized, double blinded, placebo-controlled, phase III trial of antibiotics intended to reduce MTCT of HIV-1 and preterm birth. ${ }^{8}$ Enrollment began in July 2001 and the last delivery occurred in July 2003. Recruitment into the study was stopped by the Data and Safety Monitoring Board (DSMB) in March 2003 when it became apparent that the intervention was unlikely to reduce MTCT of HIV-1, the primary objective of the study. No further antibiotics were dispensed after March 2003. This trial was conducted at 4 sub-Saharan African sites in 3 countries: Blantyre and Lilongwe, Malawi; Dar es Salaam, Tanzania; and Lusaka, Zambia. The Institutional Review Boards (IRBs) of each African and collaborating U.S. institution approved the protocol.

Within these sites, participants were recruited from multiple hospitals and antenatal clinics. All pregnant women attending their first antenatal visit were informed of the study. After administration of HIV-1 voluntary counseling and testing and confirmation of HIV-1 status, HIV-1-infected women were enrolled in the trial after an informed consent process and randomly assigned to receive either antibiotics or placebo at 20-24 weeks gestation. To reduce the stigma for the women with HIV-1, in 3 of the 4 sites, for every 5 women with HIV-1, 1 HIV-1-uninfected woman was enrolled. Dar es Salaam recruited only HIV-1-infected women.

After enrollment, follow-up visits were conducted at 26, 30, and 36 weeks, and at labor/ delivery. During these visits, women were interviewed using structured questionnaires, a physical examination was performed, and laboratory samples were obtained. Infant study visits were conducted at birth (within the first 48 hours), at 4-6 weeks (with an allowable window up to 8 weeks), and at $3,6,9$, and 12 months.

In addition to routine antenatal care, women were screened for syphilis and other genital tract infections during pregnancy and treated at no cost. All HIV-1-infected women participating in this trial and their infants were offered, and most received nevirapine according to the HIVNET 012 regimen for prevention of MTCT of HIV-1. ${ }^{9}$ Cotrimoxazole prophylaxis was given to HIV-exposed infants. HIV-1-infected women and children were referred for HIV-1 care at each of the participating study sites. However, antiretroviral drugs were not available at any of the study sites at that time. 
HIV-1 diagnostic testing of the women was performed according to site-specific procedures that included either a rapid test or ELISA/Western blot tests. All initially positive HIV-1 test results were confirmed on site. Maternal blood was collected to perform a complete blood count, CD4 cell count, and plasma viral load. Infant HIV-1 infection was determined by RNA PCR at birth, 4-6 weeks, and quarterly through 9 months and by ELISA/WB at 12 months. ${ }^{8}$ For confirmation of the infant's HIV-1 infection a second sample from a subsequent visit was tested whenever possible. Infants who were lost to follow-up or died before testing were classified as having an unknown HIV-1 infection status. Maternal plasma viral load was measured as previously described. ${ }^{8}$

\section{Adverse Event Reporting}

Adverse events (AEs) were graded according to the NIAID DAIDS Toxicity Table (http://www3.niaid.nih.gov/research/resources/DAIDSClinRsrch/PDF/Safety/ DAIDSAEGradingTable.pdf) and adapted to local population normal values including all laboratory values. For example, the definition of all grades of anemia was lower at each study site compared with the DAIDS toxicity tables. The 5 toxicity grades that were assigned to an AE were defined as follows: grade 1 (mild), grade 2 (moderate), grade 3 (severe), grade 4 (lifethreatening), and grade 5 (death). The relationship between an AE and the investigational product (nevirapine) was determined by the site investigator or subinvestigator. All AEs, regardless of their relationship to nevirapine, were entered into the study database if they occurred within 2 weeks of exposure to the study antibiotics or within 8 weeks of exposure to nevirapine. The following SAEs were reported throughout follow-up regardless of whether they took place within 8 weeks of exposure to nevirapine: death, congenital anomaly or birth defect, stillbirth, or spontaneous abortion.

\section{Statistical Analysis}

For this analysis, we define maternal SAEs as those AEs that occurred after enrollment and up to 12 months postdelivery that resulted in death, stillbirth, new or prolonged hospitalization, and AEs considered to be life-threatening (grade 4 events). Infant SAEs were defined as congenital anomalies, AEs that resulted in death, new or prolonged hospitalization, or were life-threatening (grade 4) that occurred after birth and up to 12 months of age. For HIV-1infected infants, timing of HIV-1 infection was determined by the specimen collection date of the first positive HIV-1 test, and categorized as early infection (positive results by 8 weeks of age), late infection (negative results throughout 8 weeks, followed by a positive result after week 8), and infection of unknown timing (missing test result at 4-8 weeks, and positive result after week 8). Two infants with indeterminate HIV-1 status were excluded from any analysis assessing the impact of infant HIV-1 status on SAEs or mortality. Infants with only negative test result(s) during follow-up (birth to 12 months of age) were categorized as "uninfected," although we recognize that some of the infants in this category could have been infected either after censoring or in between the last negative test and death.

Frequency tables were used to describe the distribution of SAEs and mortality by etiology. The proportions of participants experiencing SAEs or death were compared between study sites using either Pearson $\chi^{2}$ test or Fisher exact test.

Kaplan-Meier curves were used to describe infant survival time distribution by strata. Mortality rates and the corresponding 95\% confidence intervals were calculated based on the personyear analysis assuming an underlying Poisson distribution. Cox proportional hazard modeling was performed to identify maternal and infant factors associated with risk of death in the infants. Variables included in the multivariate models were based on biologic or epidemiological importance or statistical significance $(P \leq 0.05)$ in univariate analysis. 
All statistical calculation and analyses were performed using SAS version 9.1.3 on SunOS 5.9 platform.

\section{RESULTS}

\section{Size and Characteristics of the Study Population}

We enrolled 2659 women in their second trimester of pregnancy at the 4 sites between July 30, 2001 and March 5, 2003, including 2292 (86.2\%) HIV-1-infected women and 367 (13.8\%) HIV-1-uninfected women. One hundred fifty-eight HIV-1-infected women (6.9\%) and 28 HIV-uninfected women (7.6\%) were lost to follow-up before delivery. HIV-1-infected women delivered 2078 live-born infants; of these, 2026 singleton and 26 first-born twins were included in this analysis. Three hundred thirty-one HIV-1-uninfected women delivered a live-born infant of whom 327 singleton and 4 first-born twins were included in this analysis. Among these 2383 infants, $1562(66 \%)$ were followed until the end of the study (51 at 6 months, 212 at 9 months, and 1299 at 12 months of age). Three hundred forty-nine (15\%) infants died before 12 months of age. Four hundred seventy-four (20\%) infants terminated early from study participation because of relocation of the family (64\%), parents refusing further participation (32\%), or maternal death $(1.8 \%)$.

The median age among HIV-1-infected women was 25 years and the median age among HIV-1uninfected women was 22 years. The median number of years of education among both HIV-1infected and uninfected women was 7 years. There were no differences in the rate of maternal SAE and mortality, infant SAE and mortality, or infant HIV-1 infection among the randomization arms. ${ }^{8}$

\section{SAEs and Mortality Among Mothers}

Among 2292 HIV-1-infected mothers, 166 (7.2\%) had an SAE and 42 (1.8\%) died (Table 1). Thirty-five $(83 \%)$ deaths occurred after delivery and 7 (17\%) occurred before delivery. No deaths occurred during labor and delivery. The incidence of maternal SAEs and mortality did not differ among the 4 sites. Of uninfected mothers, 12 (3.3\%) experienced an SAE but none died (Table 1). Ten SAEs occurred during labor/delivery and 2 occurred before delivery. Stillbirth was the most common SAE among all mothers, comprising 37\% (77) of AEs in HIV-1-infected mothers and $67 \%$ of AEs in uninfected mothers. There was no difference in stillbirth based on baseline maternal syphilis status; stillbirth occurred in $3.7 \%$ of women positive for syphilis at baseline and in 3.2\% of women negative for syphilis at baseline $(P=$ $0.7)$. Other frequently reported SAEs among HIV-1-infected women were anemia ( $\mathrm{n}=23$, $11 \%)$, malaria $(\mathrm{n}=17,8 \%)$, and tuberculosis $(\mathrm{n}=14,7 \%)$.

Fifty-seven etiologies were attributed to the 42 deaths among HIV-1-infected mothers. Anemia $(n=11,19 \%)$, sepsis $(n=8,14 \%)$, tuberculosis $(n=6,10 \%)$, and malaria $(n=6,10 \%)$ were the most common causes of death, together accounting for about $53 \%$ of deaths. Other significant causes of death included meningitis $(n=4,7 \%)$, pneumonia $(n=5,8 \%)$, AIDSrelated complex $(n=3,5 \%)$, and Kaposi Sarcoma $(n=3,5 \%)$. The cause of death was unknown for 2 women. Among the 8 sepsis deaths, 5 occurred within 6 weeks after delivery and were possibly related to labor and delivery complications.

HIV-1-infected women with enrollment CD4 lymphocyte counts $<200$ cells $/ \mathrm{mm}^{3}$ had a higher mortality rate (6.1/100 person years, 95\% CI: 3.9 -9.1) than women with CD4 counts of 200500 cells $/ \mathrm{mm}^{3}$ (1.1/100 person years, $95 \%$ CI: $\left.0.5-1.9\right)$ or $\geq 500$ cells $/ \mathrm{mm}^{3}(0.4 / 100$ person years, 95\% CI: 0.1-1.5). Mortality was greater among women with plasma HIV-1 RNA concentrations $\geq 50,000$ copies $/ \mathrm{mL}$ (5.1/100 person years, $95 \%$ CI: $3.5-7.2)$ than among 
women with plasma HIV-1 RNA concentrations $<50,000$ copies $/ \mathrm{mL}(0.6 / 100$ person years, 95\% CI: 0.2-1.1).

\section{SAEs and Mortality Among Infants}

The infant mortality rates did not differ among the sites $(P=0.58)$. Overall, 424 infants had an SAE (17.8\%) and there were 349 deaths (14.6\%) among 2383 live-born infants followed for a median of 365 days. The proportion of infants with SAEs varied by site among infants born to HIV-1-infected women (Table 1).

Pneumonia was the most frequently reported SAE and cause of death among all infants, accounting for $22.5 \%$ of all SAEs and $30.4 \%$ of all deaths (Table 2). Other SAEs frequently experienced by all infants were sepsis, diarrhea, and malaria which together totaled $21.7 \%$ of all SAEs. Forty-nine (9.2\%) instances of sepsis were reported 14 (5.9\%) times among HIV-1infected infants and $31(12.8 \%)$ times among HIV-1-uninfected infants. Diarrhea and gastroenteritis were reported $40(7.5 \%)$ times for all infants and 25 times (10.6\%) for HIV-1infected infants and 15 times (6.2\%) among HIV-1-uninfected infants. Malaria was the etiology of $26(4.9 \%)$ SAEs (10 [4.2\%] SAEs among HIV-1-infected infants and 16 (6.6\%) SAEs among HIV-1-uninfected infants).

To assess how the timing of acquisition of HIV-1 infection affected infant mortality, deaths occurring before and after week 8 (day 56) were examined separately. By week 8, 104 (5.1\%) infants born to HIV-1-infected women died compared with 10 (3\%) infants born to HIV-1uninfected women. Among the 104 infants, 48 had no HIV-1 test before death, 9 had a positive HIV-1 diagnostic test result, and 47 had a negative test result but no positive test result before death. For infants with follow-up beyond 8 weeks of age, those who were infected early (at birth or by 4-6 weeks) experienced significantly greater mortality (Fig. 1, Table 3 ).

\section{Infant Mortality Related to Maternal HIV-1 Infection Status}

Infants born to HIV-1-infected mothers were more likely to die during the follow-up period than infants born to HIV-1-uninfected mothers (hazard ratio and 95\% CI, 3.9 [2.3, 6.9]). Additionally, infant mortality was higher among infants born to both HIV-1-infected and HIV-1-uninfected mothers who experienced an SAE (including death) than among infants whose mothers did not experience an SAE (hazard ratio and 95\% CI, 5.8 [3.5, 9.4]) (Table 4). Among infants born to HIV-1-infected women, maternal CD4 counts at enrollment were inversely related to infant mortality; compared with infants born to mothers with CD4 count $\geq 500$ at enrollment, the mortality was higher among infants born to mothers with CD4 count $<200$ (hazard ratio and 95\% CI, 3.1 [2.2, 4.2]) and infants born to mothers with CD4 count between 200 and 500 (hazard ratio and 95\% CI, 1.6 [1.2, 2.2]). Infant mortality was higher among those born to mothers with higher RNA viral loads (hazard ratio and 95\% CI for 1 unit increase in $\log 10$ viral load, 2.3 [2.0, 2.8]) (Table 4).

In multivariate analyses, maternal HIV-1 infection, morbidity, and mortality were significant predictors of infant mortality among all infants (Table 4). Among infants born to HIV-1infected mothers, maternal morbidity, mortality, baseline CD4 count, and baseline plasma HIV-1 RNA concentration were all significant predictors of infant mortality (Table 4).

\section{DISCUSSION}

This multisite study was conducted in Africa at a time when there were few opportunities to access ART or adequate care for opportunistic infections. Since late 2003, national programs have scaled up and now provide ART to large numbers of infected individuals, dramatically 
improving the survival and quality of life for those involved. These data describe the natural history of HIV disease in antenatal women and their infants before ART availability.

In this study, mortality among mothers and infants did not differ among sites.

Two percent of the HIV-infected women died during the follow-up period that began in their second trimester of pregnancy and continued for 12 months after delivery. There were no deaths among the 367 HIV-negative women and only $3 \%$ of these women had a SAE, illustrating the differences between HIV uninfected and infected women. Each of the countries where the study was conducted have some of the highest maternal mortality ratios in the world: Malawi 984 per 100,000 live births, ${ }^{10}$ Tanzania 578 per 100,000 live births, ${ }^{11}$ Zambia 729 per 100,000 live births. ${ }^{12}$ However, although the majority of SAEs occurred during labor and delivery for all women, $90 \%$ of deaths occurred postnatally and no deaths occurred during labor and delivery. These findings suggest that the high maternal mortality reported in these countries may be more directly influenced by the underlying health status, such as HIV-status and anemia.

The primary cause of death among these women was anemia. The prevalence of anemia is high among women in sub-Saharan Africa. National population-based surveys estimate $44 \%$ of women in Malawi and $48 \%$ of women in Tanzania are anemic. ${ }^{10,11}$ Anemia is a leading contributor to maternal morbidity and mortality in developing countries ${ }^{13}$ and is caused primarily by malaria, intestinal parasites and HIV itself. Our results support the role of reducing anemia in women to reduce maternal mortality, especially among HIV-infected women.

Infant mortality, with or without HIV infection, is high in these countries. In contrast to the maternal cohort in which only $25 \%$ of SAEs resulted in death, the majority of SAEs (82\%) among the infants resulted in death. Mortality was high in this cohort of infants where $86 \%$ of the mothers were HIV-infected and 20\% of the infants were HIV infected by the end of the 12 month follow-up period. Almost $15 \%$ of the infants in the cohort died during this follow-up period (146 per 1000 live births), higher than the all infant mortality in Tanzania (68 per 1000 live births), ${ }^{11}$ Zambia (95 per 1000 live births), ${ }^{12}$ and Malawi (76 per 1000 live births). ${ }^{10}$ Mortality among HIV-1-infected infants was more than 5 times as high as uninfected infants regardless of the timing of infection. By the end of the follow-up period, $42 \%$ of infected infants had died, compared with $7.2 \%$ of uninfected HIV-exposed infants and $4.8 \%$ of HIV unexposed infants. The mortality was the highest among infants infected early (at birth or 4-6 weeks) and confirms other studies of perinatal transmission. ${ }^{14,15}$ The mortality rate among infants infected early is more than 3 -fold higher than the mortality rate of infants infected after 8 weeks. This high mortality underscores the importance of early diagnosis and immediate referral to ART. Increasing access to appropriate therapy is essential to prevent early mortality. ${ }^{16}$

Infant outcomes were strongly related to maternal outcomes. Infants in our study were significantly more likely to die if their mother experienced an SAE or died, regardless of the infant's HIV status. In univariate analysis, the mortality rate was more than 2-fold higher among infants born to mothers who experienced an SAE and >4-fold higher among infants born to mothers who died. Among the HIV-infected mothers, the relationship of the mother's health to the outcome of the infant can also be quantified by the maternal CD4 count and the HIV viral load at baseline. The lower the mother's CD4 count or the higher the viral load at enrollment, the more likely the infant was to die in the first year. An infant of a mother with a CD4 count $<200$ was 3 times more likely to die than a child of a mother with a CD4 count $>500$. All the unadjusted maternal factors in the analysis of infant mortality continued to be significant in the multivariate analysis including HIV infection, maternal morbidity and mortality, CD4 count, and RNA viral load. Clinical diagnosis or WHO staging will not 
accurately identify all those with a low CD4 count. Therefore, CD4 count should be included as part of routine prenatal HIV testing after post-test counseling of all infected women.

The limitations of this study include the underestimation of HIV-related morbidity and mortality among infants. The HIV-status was not determined at death and some of these infants may have been infected after their last negative test. Because of the testing sequence, an infant with a negative HIV-test at birth and at 6-8 weeks who subsequently seroconverted and died before the next follow-up visit would be classified as HIV-negative or unknown. Mothers or infants receiving care elsewhere for an SAE were not captured by this analysis. Additionally, data from 3 sites with diverse populations were pooled for this analysis and differences may exist in standard of care, protocols for making clinical diagnoses, care-seeking behavior, and access. However, these differences are not likely to be significant as our analysis demonstrated similar outcomes in all 3 populations. Also, the exclusion criteria for this study selected for asymptomatic women and excluded the symptomatic or chronically ill. This likely reduced the maternal morbidity and mortality in this study population relative to a population-based sample.

Because of the significant difference in mortality of infants born to HIV-infected compared with uninfected mothers, the counseling message to all HIV-positive women should state this increased risk of death to a newborn. This message will help women make informed reproductive decisions. Additional programs should provide early and routine HIV testing for all pregnant women, CD4 monitoring at the time of testing for infected mothers, early diagnosis, and appropriate early treatment for HIV-exposed infants, a family support program for infants of sick or deceased mothers, and appropriate and timely ART for infected mothers and infants, including ART regimens for the PMTCT.

\section{Acknowledgments}

This study was supported by the HIV Network for Prevention Trials (HIVNET) and sponsored by the U.S. National Institute of Allergy and Infectious Diseases (NIAID), the National Institutes of Health, and Department of Health and Human Services, through contract \#N01-AI-35173 with Family Health International; contract \#N01-AI-45200 with Fred Hutchinson Cancer Research Center; and subcontract \#N01-AI-35173-117/412 with Johns Hopkins University. This work was also sponsored by the U.S. National Institute of Allergy and Infectious Diseases, National Institute of Child Health and Human Development, National Institute on Drug Abuse, National Institute of Mental Health, and the Office of AIDS Research at the National Institutes of Health, U.S. Department of Health and Human Services, Harvard University (U01-AI-48006), Johns Hopkins University (U01-AI-48005), and the University of Alabama at Birmingham (U01-AI-47972). Nevirapine (Viramune, Boehringer Ingelheim GmbH, Ingelheim, Germany) for the study provided by Boehringer Ingelheim Pharmaceuticals, Incorporated.

\section{REFERENCES}

1. UNAIDS. AIDS Epidemic Update, December 2005. 2006

2. Ferradini L, Jeannin A, Pinoges L, et al. Scaling up of highly active antiretroviral therapy in a rural district of Malawi: an effectiveness assessment. Lancet 2006;367:1335-1342. [PubMed: 16631912]

3. Harries AD, Schouten EJ, Libamba E. Scaling up antiretroviral treatment in resource-poor settings. Lancet 2006;367:1870-1872. [PubMed: 16753490]

4. National Scaling up of ARV therapy in Malawi. The HIV Unit Ministry of Health: Malawi Ministry of Health; 2004.

5. Weller I. Delivery of antiretroviral therapy in sub-Saharan Africa. Clin Infect Dis 2006;43:777-778. [PubMed: 16912955]

6. Stringer JS, Zulu I, Levy J, et al. Rapid scale-up of antiretroviral therapy at primary care sites in Zambia: feasibility and early outcomes. JAMA 2006;296:782-793. [PubMed: 16905784]

7. Goldenberg RL, Mudenda V, Read JS, et al. HPTN 024 study: histologic chorioamnionitis, antibiotics and adverse infant outcomes in a predominantly HIV-1-infected African population. Am J Obstet Gynecol 2006;195:1065-1074. [PubMed: 16875654] 
8. Taha TE, Brown ER, Hoffman IF, et al. A phase III clinical trial of antibiotics to reduce chorioamnionitis-related perinatal HIV-1 transmission. AIDS 2006;20:1313-1321. [PubMed: 16816561]

9. Guay LA, Musoke P, Fleming T, et al. Intrapartum and neonatal single-dose nevirapine compared with zidovudine for prevention of mother-to-child transmission of HIV-1 in Kampala, Uganda: HIVNET 012 randomised trial. Lancet 1999;354:795-802. [PubMed: 10485720]

10. Malawi Demographic and Health Survey 2004. National Statistical Office (Malawi) and ORC Macro; Calverton, MD: 2005.

11. Tanzania Demographic and Health Survey 2004-05. National Bureau of Statistics and ORC Macro; Dar es Salaam, Tanzania: 2005.

12. Zambia Demographic and Health Survey 2001-2002. Central Statistical Office (Zambia), Central Board of Health, and ORC Macro; Calverton, MD: 2003.

13. Rush D. Nutrition and maternal mortality in the developing world. Am J Clin Nutr 2000;72(1 Suppl): 212S-240S. [PubMed: 10871588]

14. Newell ML, Coovadia H, Cortina-Borja M, Rollins N, Gaillard P, Dabis F. Mortality of infected and uninfected infants born to HIV-infected mothers in Africa: a pooled analysis. Lancet 2004;364:12361243. [PubMed: 15464184]

15. Zaba B, Whitworth J, Marston M, et al. HIV and mortality of mothers and children: evidence from cohort studies in Uganda, Tanzania and Malawi. Epidemiol 2005;16:275-280.

16. Aledort JE, Ronald A, Le Blancq SM, et al. Reducing the burden of HIV/AIDS in infants: the contribution of improved diagnostics. Nature 2006;444(suppl 1):19-28. [PubMed: 17159891] 


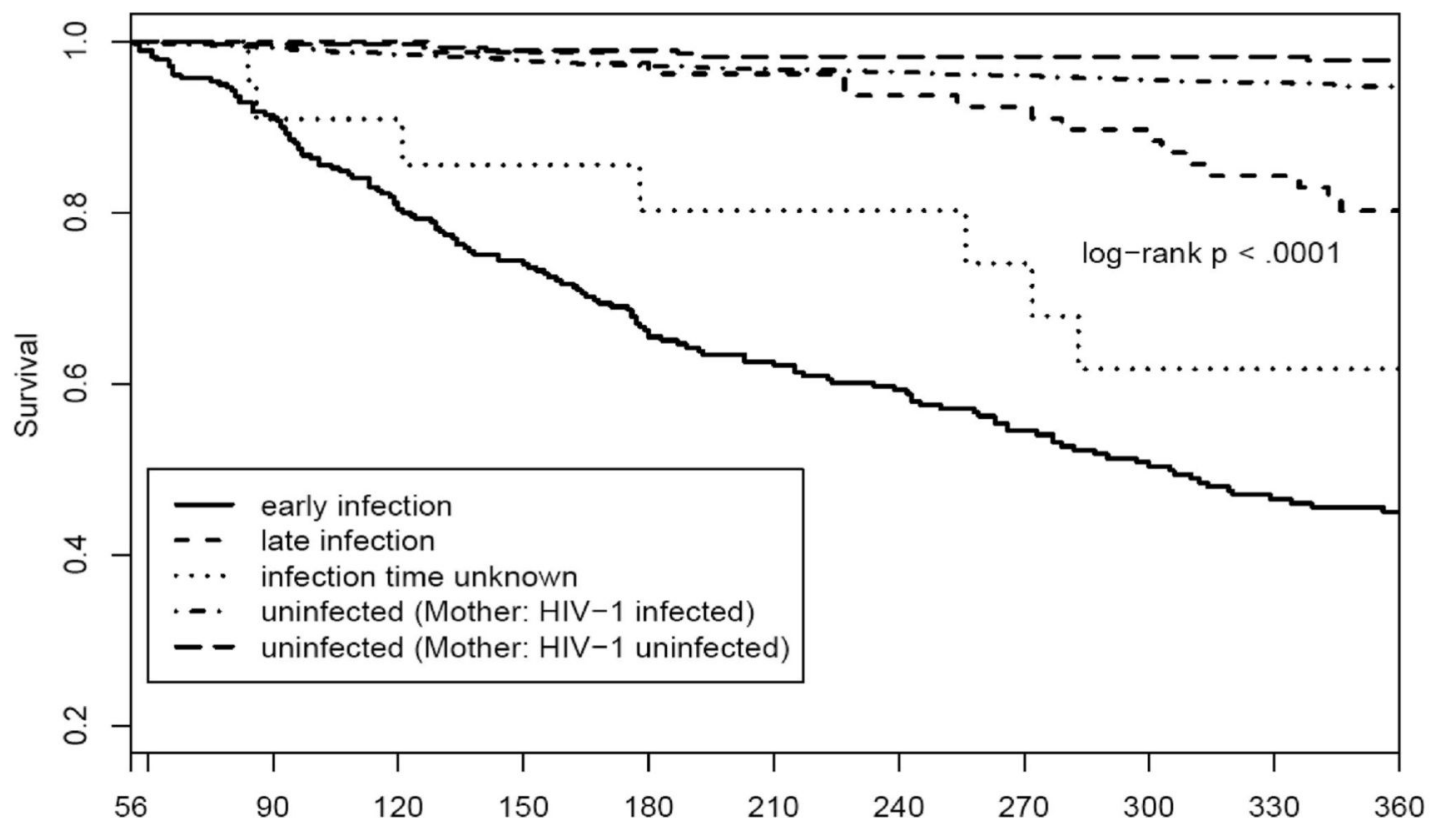

FIGURE 1.

Infant survival by timing of HIV infection. 


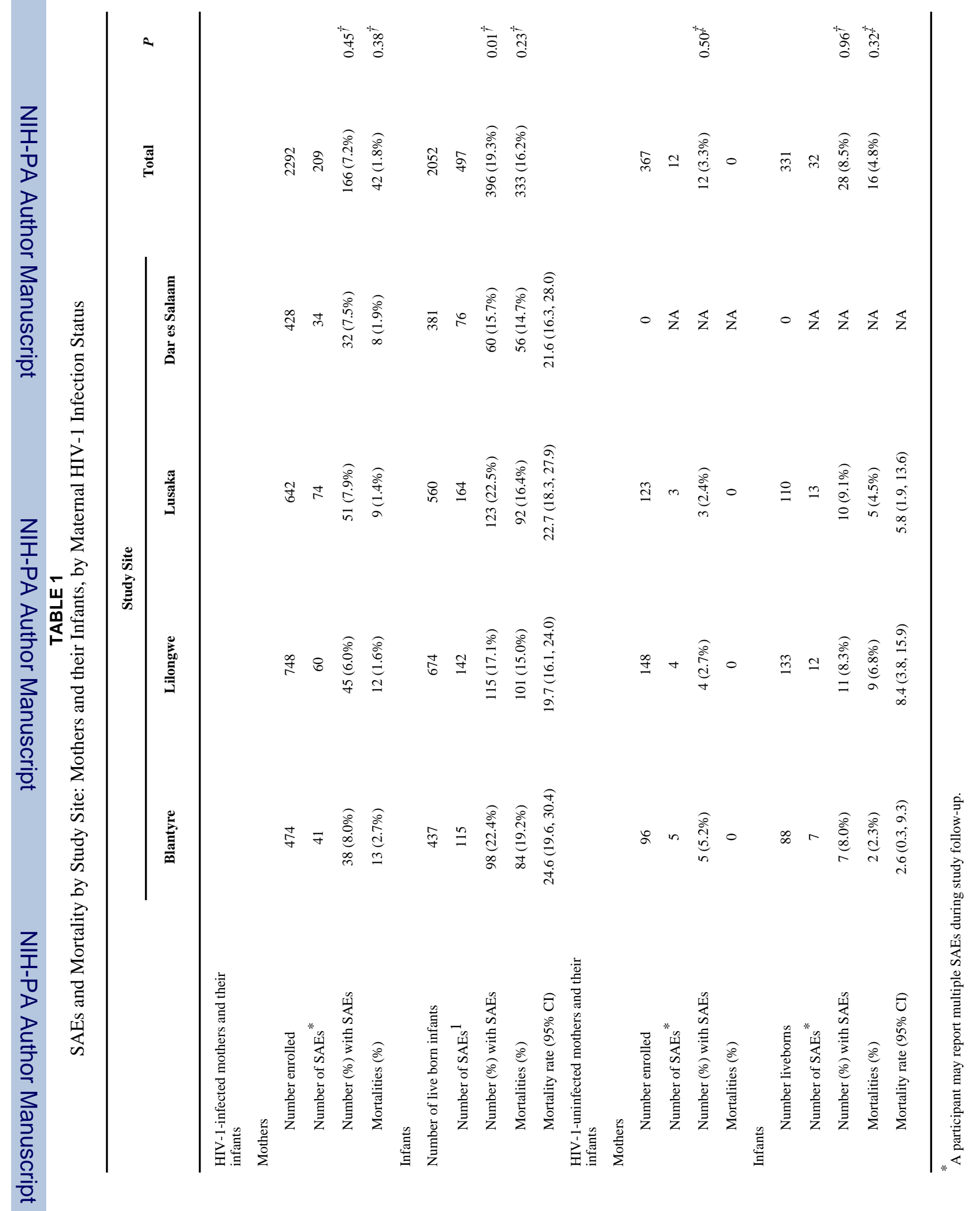


TABLE 2

Causes of Infant Mortality, Overall and by Infant HIV-1 Infection Status

\begin{tabular}{|c|c|c|c|}
\hline Cause of Mortality & $\begin{array}{c}\text { HIV-1-Infected } \\
\text { Infants }(\mathrm{N}=404) \\
\text { Number of Deaths }(\%)\end{array}$ & $\begin{array}{c}\text { HIV-1-Uninfected } \\
\text { Infants }(\mathrm{N}=1904) \\
\text { Number of Deaths }(\%)\end{array}$ & $\begin{array}{c}\text { All Infants } \\
(\mathbf{N}=2383) \\
\text { Total Deaths }(\%)\end{array}$ \\
\hline Pneumonia & $72(17.8)$ & $34(1.8)$ & $106(4.4)$ \\
\hline Diarrhea/gastroenteritis & $26(6.4)$ & $16(0.8)$ & $42(1.8)$ \\
\hline Unknown cause & $15(3.7)$ & $15(0.8)$ & $36(1.5)$ \\
\hline Sepsis & $10(2.5)$ & $21(1.1)$ & $35(1.5)$ \\
\hline Malaria & $10(2.5)$ & $15(0.8)$ & $25(1.0)$ \\
\hline Oral candidiasis & $10(2.5)$ & $3(0.2)$ & $13(0.5)$ \\
\hline Tuberculosis & $8(2.2)$ & $0(0)$ & $9(0.4)$ \\
\hline Other & 49 (11.9) & $53(2.8)$ & $142(6.0)$ \\
\hline Total causes & 200 & 157 & 408 \\
\hline Total deaths & $170^{\dagger}$ & $130^{\dagger}$ & $349^{\dagger+}$ \\
\hline
\end{tabular}




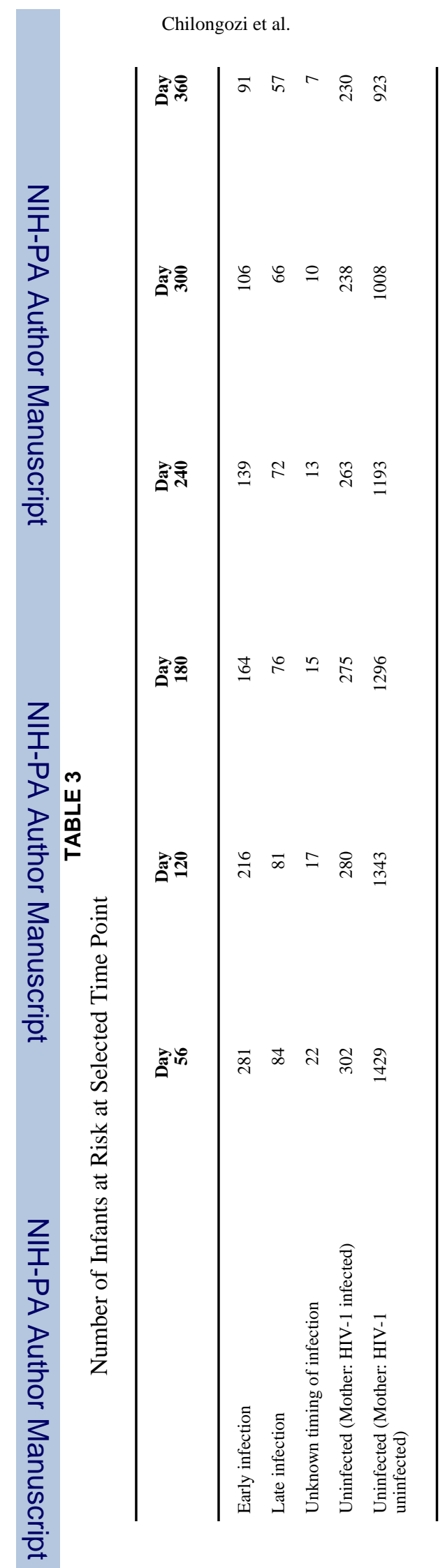

Page 14 


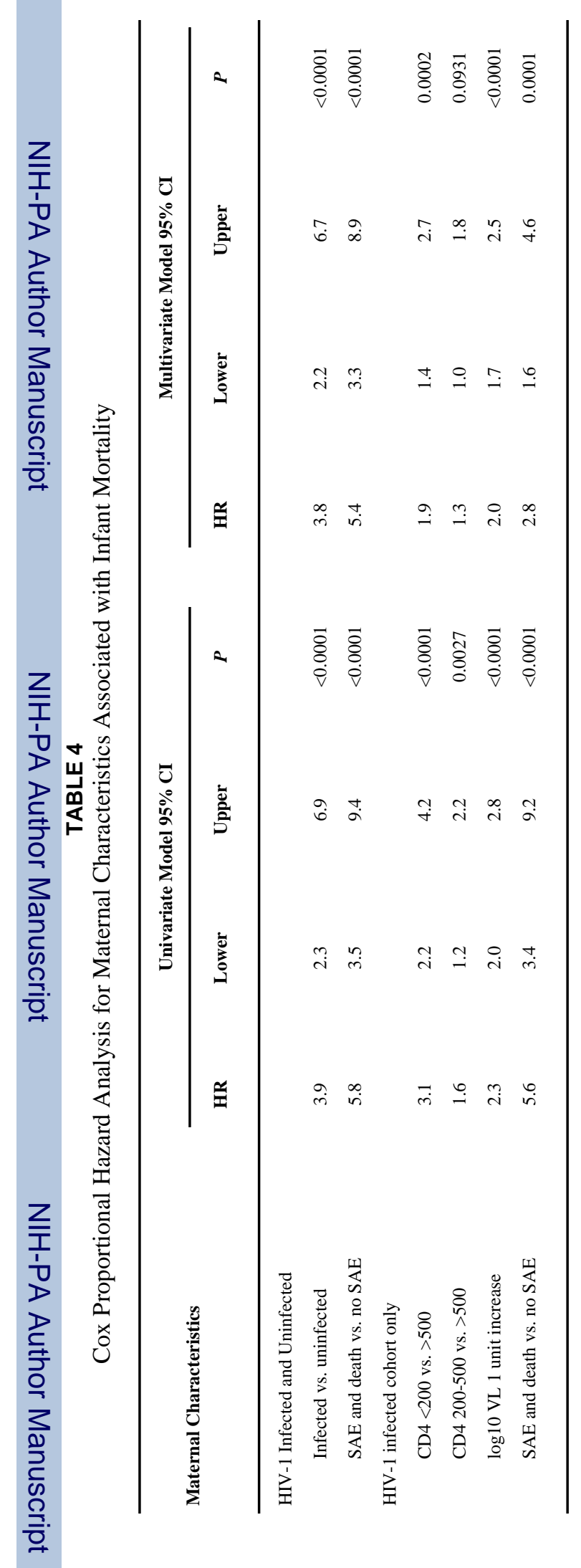

Pediatr Infect Dis J. Author manuscript; available in PMC 2009 September 8. 